\title{
Diffusion-based Non-uniform Regularization for Variational Shape Deformation
}

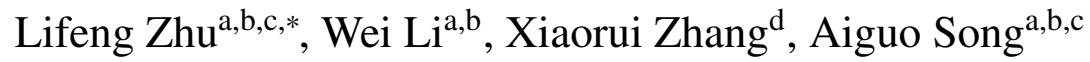 \\ ${ }^{a}$ School of Instrument Science and Engineering, Southeast University \\ ${ }^{b}$ Jiangsu Province Key Lab. of Remote Measuring and Control \\ ${ }^{c}$ CICAEET, Nanjing University of Information ScienceE Technology \\ ${ }^{d}$ School of Computer and Software, Nanjing University of Information ScienceE Technology
}

\begin{abstract}
Regularization is a typical technique to correct the discontinuity artifacts at the control points in shape deformation. A regularizer with higher weights is required if the deformation is large, which will unfortunately distort the entire shape. In this work, we present a non-uniform regularization technique based on a shape-aware scalar field obtained from diffusion, which allows user to control the magnitude and range of the regularizer around specific control points. Experimental results show that shapes are deformed smoothly and no over-regularized artifact is observed with our non-uniform regularizer.
\end{abstract}

(C) 2011 Published by Elsevier Ltd.

Keywords:

Regularization, Variational Deformation, Diffusion

\section{Introduction}

Shape deformation is an important tool for shape design and computer animation. It is studied by a range of prior work. Among all the techniques, variational shape deformation which minimizes quadratic energies once [1] or iteratively [2] is popular, because of its simplicity in the formulation and efficiency during the computation. It is also flexible to control the deformation by adding linear constraints to the system, if we provide an intuitive and flexible interface to the users. However, it is prone to discontinuity artifacts at the control points when the linear constraints are modeled as interpolatory constraints. Since the gradients of the deformation energy sharply increase around the control points, flipped triangles or highly distorted elements will appear after the global solve, as shown in Fig. 1(b).

One way to avoid the discontinuity artifacts is to use nonlinear deformation energies or adding nonlinear constraints to the system, at the cost of the computational efficiency. Reg- ${ }^{21}$ ularization is another preferred technique to suppress the arti- ${ }^{22}$ facts. Basically, a quadratic regularizer is sufficient to remove ${ }^{23}$

${ }^{*}$ Dr., Corresponding author

Email address: lfzhulf@gmail.com (Lifeng Zhu)

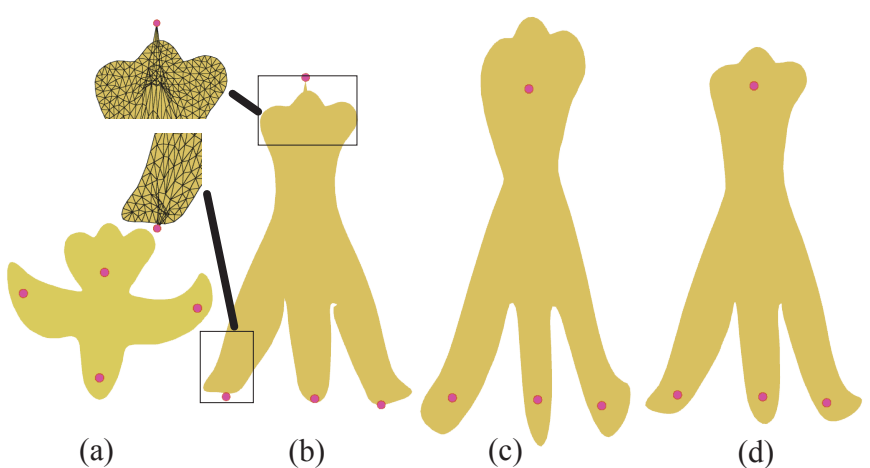

Figure 1. (a)The undeformed shape and control points. (b)The deformed shape without regularization. (c)The deformed shape with strong uniform regularization. (d)The deformed shape with our non-uniform regularization. The as-rigidas-possible (ARAP) energy is used and close-up views are shown to highlight the artifacts.

the discontinuity artifacts while no significant runtime overhead is expected.

Although it is effective to uniformly blend the deformation energy term and regularizer, extra distortion might be introduced around the area far from the control points. Especially when the regularizer is strong, the distortion on the over- 
regularized shape is observable, as shown in Fig. 1(c). We 81 usually have to trade off the smoothness and the distortion of 82 the deformed shape. However, for applications such as indus- 83 trial design, both the smoothness and distortion of the shape 84 are essential and it is sometimes hard to find a good trade-off. 85 We therefore propose to use non-uniform regularization to im- 86 prove the quality of the deformed shape. Specifically, a spatial- 87 varying scalar field on the undeformed shape is computed by 88 using diffusion-based methods. The scalar field is used to char- 89 acterize the amount of the regularization, which prefers strong 90 regularizer around the control points. Two extra parameters de- 91 fined on each control point are introduced to control the mag- 92 nitude and range of the local regularization. The effect of our 93 non-uniform regularization can be seen in Fig. 1(d).

In the following sections, we will briefly review the related works in Sec. 2 first. Then how we formulate the non-uniform regularization is introduced and discussed in Sec. 3. Experi- ${ }^{95}$ ments are finally designed to validate our method in Sec. 4.

\section{Related Work}

Shape deformation is broadly studied recently. In this work, we focus on variational shape deformation, which usually does not require users to embed cages or skeletons on the shape. In variational shape deformation, measurements are designed to quantify the distortion of the shape and the deformation is defined to minimize the distortion. A comprehensive survey of linear variational shape deformation can be found in [1]. A few more distortion measurements are summarized in [3] [4]. Recently, subspace deformation is introduced to generate deformation without discontinuity artifacts [5] [6], which requires to first rig the shape and construct a subspace of smooth deformation. It is also shown that carefully-designed nonlinear distortion measurements may not lead to discontinuity artifacts near the control points [3] [7]. It is also possible to remove the discontinuity artifacts by introducing extra nonlinear con- 97 straints to the variational system [8] [9] [10]. Although non- 98 inversion is globally guaranteed in the above-mentioned work 99 if the problem is feasible, it is relatively expensive to compute 100 the deformation by using these nonlinear methods.

Regularization is another simple yet effective technique to suppress the discontinuity artifacts. By increasing the weights of the regularizer, smoother deformation around the control points is produced. Making the positional constraints soft is the most widely used way to prevent the artifacts. It could also be seen ${ }^{10}$ as a special regularizer to the variational problem. The standard ${ }^{102}$ Tikhonov regularization tries to regularize the solution by $\mathrm{di}^{-{ }^{103}}$ rectly enforcing the smoothness of the deformation, which will ${ }^{104}$ unfortunately shrink the result a little. Recently, a quadratic ${ }^{105}$ problem-specific regularizer is proposed in [4]. Martinez Es- ${ }^{106}$ turo et al. show that the artifacts around control points are ef- ${ }^{107}$ fectively smoothed out by adding their regularizer to various ${ }^{108}$ deformation problems. Later the regularizer is improved to be ${ }^{109}$ curvature-aware in [11]. A common problem with regulariza- ${ }^{110}$ tion is that the solutions may be spoiled if the amount of reg- ${ }^{111}$ ularization is too high. In this work, we are trying to reduce ${ }^{112}$ the amount of regularization while preventing the discontinuity artifacts.

Diffusion is a natural phenomenon which produces smooth and time-varying distributions. It has been introduced to geometric processing since the last century. In the early work on surface fairing [12], diffusion has been shown to be effective to smooth out levels of geometric noises. Diffusion is also related to intrinsic shape features [13] [14]. Recently, more applications of diffusion-based techniques in geometric processing have been found. To name a few, diffusion models were used to compute approximate geodesics [15], shape segmentation [16], shape matching [17] and medial axis [18]. In this work, we will use diffusion-based techniques to control the regularizer for shape deformation.

\section{Method}

\subsection{Background and Notations}

We will first define our notations and briefly review the most recent related techniques in this subsection. Suppose the input undeformed shape is discretized as a triangular mesh $(V, F)$ with $n$ vertices and $m$ faces, where $V=\left[\overline{\mathbf{v}}_{1}, \overline{\mathbf{v}}_{2}, \ldots, \overline{\mathbf{v}}_{n}\right]$ stores the undeformed geometry of the shape and $F=\left[\mathbf{t}_{1}, \mathbf{t}_{2}, \ldots, \mathbf{t}_{m}\right]$, in which $\mathbf{t}_{i}=\left[i_{1}, i_{2}, i_{3}\right]^{\prime}$ stores the vertex indices of the $i$ th triangle. To deform the rest shape, the users are then required to specify a subset of the vertices $\mathcal{J}$ as the control points and their deformed position $\mathbf{v}_{j}(j \in \mathcal{J})$. In order to enforce those interpolatory constraints, the deformed control points are modeled as hard constraints, i.e., they are eliminated from the system in this work. In variational shape deformation, the unknown position of deformed vertices $\mathbf{v}$ is defined to minimize the distortion of the mesh

$$
D(\mathbf{v})=\sum_{i=1}^{m} a_{i} D_{i}(\mathbf{v})
$$

under the given deformation configuration, where $D_{i}(\mathbf{v})$ measures the distortion of the $i$ th face and $a_{i}$ weights the contribution of $i$ th face. A typical choice of $a_{i}$ is the area of the $i$ th face.

As-rigid-as-possible (ARAP) energy is a popular choice of the distortion measurement [19] [20], which can be written as

$$
D_{i}(\mathbf{v})=\left\|F_{i}(\mathbf{v})-R_{i}(\mathbf{v})\right\|_{F}^{2}
$$

where $F_{i}(\mathbf{v})$ is the deformation gradient $\partial \mathbf{v} / \partial \overline{\mathbf{v}}$ of the $i$ th face, $R_{i}(\mathbf{v})$ is the rotational component of $F_{i}(\mathbf{v})$ and $\|\cdot\|_{F}$ is the Frobenius norm. In the discrete case, the deformation gradient $F_{i}(\mathbf{v})$ is represented as the linear transformation that maps the $i$ th rest triangle to its deformed shape. The rotation $R_{i}(\mathbf{v})$ is computed from the polar decomposition of the $F_{i}(\mathbf{v})$. The nonlinear nature of the ARAP measurement ensures the quality of the deformation. Meanwhile, the nonlinear polar decomposition is only computed locally, which makes it practical in shape deformation.

In the following, we will use ARAP energy to demonstrate our method. In the variational shape deformation framework, 
there are other quadratic measurements which well approximate the ARAP energy. If the nonlinear rotational component $R_{i}(\mathbf{v})$ is defined to be the identical transformation, $D_{i}(\mathbf{v})$ turns to be the Dirichlet energy. If $R_{i}(\mathbf{v})$ is replaced by a similar transformation, the deformation from minimizing $D(\mathbf{v})$ is as-similaras-possible (ASAP). Martinez Esturo et al. [4] use the first order Taylor expansion of $R_{i}(\mathbf{v})$ around the identical transformation to approximate the rotational component, which gives a linearized ARAP version of $D_{i}(\mathbf{v})$, while Igarashi et al. [21] use the rotational component extracted from ASAP to approximate $R_{i}(\mathbf{v})$. These linear approximations lead to faster computation, but they all sacrifice the quality of the deformation to some extent comparing with the ARAP deformation. Although there are other nonlinear measurements which better describe the distortion, we do not use them in this work because they require to solve relative expensive nonlinear systems.

Because minimizing ARAP or other quadratic energies may cause strong local energy variation around the control points, the deformed shape exhibits various artifacts near control points. ${ }^{165}$ In order to smooth out the artifact, a quadratic regularizer which ${ }^{166}$ prefers the smoothness of the energy distribution is recently ${ }^{167}$ proposed in [4]. The regularizer can be written as

$$
S(\mathbf{v})=\sum_{e_{k l}} S_{k l}(\mathbf{v})=\sum_{e_{k l}} c_{k l}\left\|D_{k}(\mathbf{v})-D_{l}(\mathbf{v})\right\|^{2},
$$

in which it sums up the difference of the energy of neighbor-172 ing faces over all the interior edges $e_{k l}$ which separates the $k$ th $^{173}$ and $l$ th triangle. $c_{k l}$ weights the difference and can be set as 174 the length of the edge $e_{k l}$. A global parameter $\beta$ is then intro-175 duced to control the amount of regularization. Specifically, the176 deformed geometry $\mathbf{v}$ is determined by minimizing

$$
E(\mathbf{v})=(1-\beta) D(\mathbf{v})+\beta S(\mathbf{v})
$$

Martinez Esturo et al. shows that the regularization with $S(\mathbf{v})_{180}$ outperforms standard Tikhonov regularization which smoothes the geometry of the shape instead of the energy distribution ${ }_{{ }_{181}}$ Therefore, we use this energy-specific regularizer in the following. We only listed the notations and formulas for triangu- ${ }^{-182}$ lar meshes here. The extension to tetrahedral meshes is similar ${ }_{183}$ and we will only use triangular meshes to demonstrate our tech-184 nique in this work.

\subsection{Diffusion-based Weighting Scheme}

As shown in Fig. 1(c), the deformed shape may be overregularized if the regularizer is globally applied to the varia- ${ }^{189}$ tional deformation system. Therefore, we ask ourselves whether it is possible to reduce the amount of regularization while $\mathrm{no}_{190}$ discontinuity artifacts are noticeable. In (2), the amount of ${ }_{191}$ the regularization is only controlled by one uniform parameter ${ }_{192}$ $\beta$, which is not flexible to respond the locality of the discon- ${ }_{193}$ tinuity artifacts. To increase the flexibility, we propose to use ${ }_{194}$ a non-uniform scalar field defined on the rest shape to guide ${ }_{195}$ the amount of regularization on the shape, which is denoted by $y_{196}$ $\beta(\overline{\mathbf{v}})$ hereafter. In the discrete case, the entire system turns to ${ }_{197}$ minimize

$$
E(\mathbf{v})=\sum_{i=1}^{m}\left(1-\beta_{i}\right) a_{i} D_{i}(\mathbf{v})+\sum_{e_{k l}} \beta_{k l} S_{k l}(\mathbf{v})
$$

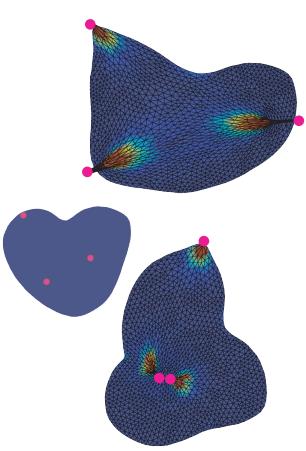

(a)

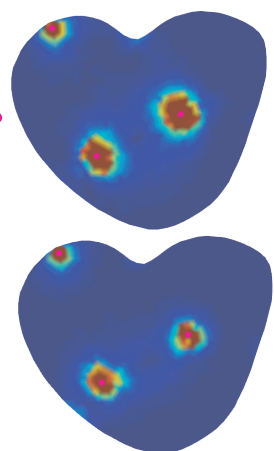

(b)

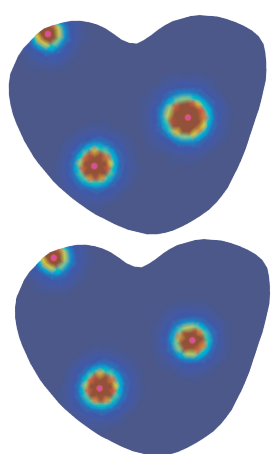

(c)
Figure 2. The smoothness energy of the distortion is used to visualize the discontinuity artifacts (b). It is high around the control points for various deformation configurations (a). Two scalar fields generated from heat diffusion (c) well match the distribution of discontinuity up to a scale.

where $a_{i}$ is the area of the $i$ th face, $\beta_{i}$ and $\beta_{k l}$ weight the amount of regularization and they will be defined in the following subsections.

Because the regularizer (1) is trying to penalize the nonsmoothness of the energy distribution, it should be negligible if the un-regularized energy distribution is smooth. Therefore, the regularization is sufficient if $\beta(\overline{\mathbf{v}})$ well fits the distribution of the energy smoothness. In this case, we test the deformed shape without regularization and visualize the smoothness of the distortion $S_{k l}(\mathbf{v})$ in Fig. 2. From the test, we observe that the heat distribution diffused from the control points well approximates the smoothness error of the energy around the control points for various deformation configurations. Therefore, we use a diffusion-based model to formulate our weighting scheme $\beta(\mathbf{v})$. Specifically, $\beta(\mathbf{v})$ is modeled to be the solution of the following diffusion equation

$$
\frac{\partial \mathbf{x}}{\partial t}=\Delta \mathbf{x}
$$

As the discontinuity artifacts are significant around the control points, we will apply stronger regularizer near the control points. In this way, we add Dirichlet boundary conditions to eqn. (4) to make the weights at the control points to be 1, i.e., $\beta\left(\overline{\mathbf{v}}_{j}\right)=1$, for every $j \in \mathcal{J}$ at any time $t$. So the weights comply with a diffusion equation with thermostatic sources located at the control points. The initial weights $\mathbf{x}_{t_{0}}$ are set to zero except for the control points.

\subsubsection{Discretization}

In order to feed the weights to (3), we have to solve the equation (4) on a triangular mesh. In the spatial discretization, we first solve the eqn. (4) at each vertex for the diffused weights $\mathbf{x}$, which is now a $n \times 1$ vector. As recommended in [22], we use the cotangent formula to represent the discrete version of the Laplacian Operator $\Delta$ in eqn. (4). We assume the cotangent Laplacian is encoded into a $n \times n$ matrix $L$. For the detailed formulation of the cotangent Laplacian, we refer to [23] and the third chapter of [22]. 

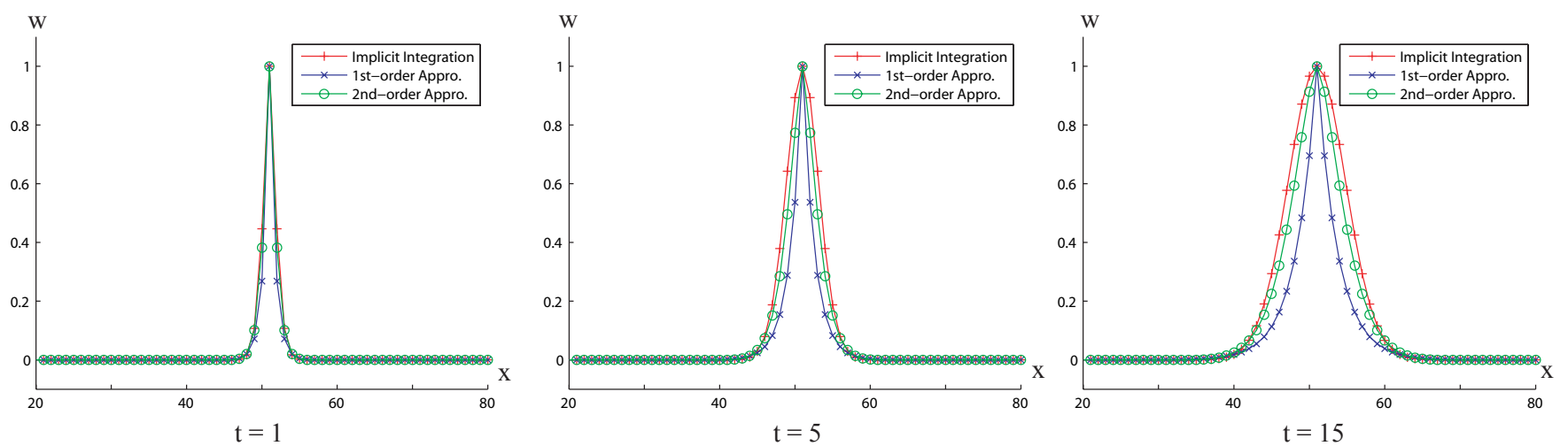

Figure 3. From left to right: the diffused weights by solving eqn. (6), (7) and (8) at $t=1,5$ and 15 , respectively. The heat source is located at $x=50$ and the time step $h$ is set to 0.0001 in this test.

For the temporal discretization of (4), we use the backward ${ }_{217}$ Euler method as proposed in [12]. Specifically, we have $\quad{ }_{218}$

$$
(I-h L) \mathbf{x}_{t_{n+1}}=\mathbf{x}_{t_{n}}
$$

where $I$ is the identity matrix, $\mathbf{x}_{t_{n}}$ is the diffused distribution at 220 time $t_{n}$ and $h$ is the time step such that $t_{n+1}=t_{n}+h$. Therefore, if the time step $h$ is tiny, the diffused distribution $\mathbf{x}_{t_{n}}$ at time $t_{n^{221}}$ satisfies

$$
(I-h L)^{n} \mathbf{x}_{t_{n}}=\mathbf{x}_{t_{0}}
$$

This implicit formula is unconditionally stable comparing ${ }^{225}$ with explicit forward Euler method [12]. Although the size ${ }^{226}$ of the time step $h$ does not affect the stability in solving the ${ }^{227}$ diffusion equation, we do prefer smaller time step $h$ because $\mathrm{e}^{228}$ the precision of the solution is higher with a high-resolution ${ }^{229}$ discretization in the time domain.However, with smaller time ${ }^{230}$ step $h$, the number of the steps $n$ increases for a given time $t_{n}^{231}$ and it takes time to get the diffused distribution. For large $t_{n}, \mathrm{it}^{232}$ requires to solve eqn. (5) $n$ times. Although we can prefactorize ${ }^{233}$ $I-h L$, it still takes time. In our implementation, we simplify ${ }^{234}$ eqn. (6) to get its approximate solution. Note that the Taylor ${ }^{235}$ expansion of $(I-h L)^{n}$ at $I$ is

$$
(I-h L)^{n}=I-n h L+\frac{n(n-1)}{2} h^{2} L^{2}+\ldots
$$

We ignore the higher order terms of its Taylor expansion $\operatorname{and}^{240}$ eqn. (6) turns to be

$$
(I-n h L) \mathbf{x}_{t_{n}}=\mathbf{x}_{t_{0}}
$$

Note that $t_{n}=t_{0}+n h$ and suppose $t_{0}=0$, the above equation 245 can be written as

$$
(I-t L) \mathbf{x}_{t}=\mathbf{x}_{0}
$$

As discussed above, we eliminate the weights at the control ${ }^{249}$ points which are set to be 1 as the boundary condition. The initial distribution $\mathbf{x}_{0}$ is set to be zero except the control points. ${ }^{250}$ Then we solve eqn. (7) once to get the approximate diffused ${ }^{251}$ weights $\mathbf{x}_{t}$ at a given time $t$. The eqn. (7) also relates to the252 screened Poisson equation and can be used for mesh denoise ${ }^{253}$
[24]. In this work, we regard it as an approximate discretization of the diffusion equation (4). We also propose to use the simplified equation with second order precision

$$
\left(I-t L+\frac{t^{2}}{2} L^{2}\right) \mathbf{x}_{t}=\mathbf{x}_{0} .
$$

Note that, by expanding eqn. (6), we have another term $-t h L^{2} / 2$ in the second order approximation. We omit this term in our approximation since we assume the smaller time step $h$. In this case, the time step $h$ is dropped in our system. Instead, we will use the parameter $t$ in the following. To illustrate the effect of the approximation, we compute the diffused weights on onedimensional domain, as shown in Fig. 3. From the test, we observe that the time parameter $t$ roughly controls the smoothness of the diffused weights. The diffused weights will have a smoother distribution if the parameter $t$ is larger. Another observation is that the diffused weights from higher order approximation (7) are smoother than those from lower order approximation, which well match the diffused weights computed from implicit integration (6) when $t$ is larger.Therefore, we choose to solve eqn. (7) when $t$ is small since the matrix $I-t L$ has less non-zero elements and thus it is more efficient to solve. If more computational resource is available, we propose to solve the weights from the eqn. (8) because it has better precision to approximate the diffusion for larger $t$. In our application, we found it is sufficient to use the approximate diffused weights from the eqn. (8) for various time parameters.

There are alternative choices for the temporal discretization of (4) such as various ways to compute matrix exponentials. We use (7) or (8) to get the approximate solution due to its simplicity. Besides, it is also easy to plug our boundary conditions into (7) or (8).

Having got the vertex weights $\mathbf{x}_{t}$ at time $t$, we finally set the edge weights $\beta_{k l}$ and face weights $\beta_{i}$ in (3) to be the average weights of their incident vertices.

\subsubsection{Properties and Discussion}

In this subsection, we will discuss about why we choose the diffusion-based weighting scheme. We will first list several preferable properties of the diffused weights: 
- Multi-scale. The range of the diffused weights monotonically increases with the time $t$ increases [13]. With smaller $t$ the distribution of weights is highly concentrated around the control points while larger $t$ will lead to near-uniform weights. When the time $t$ approximates infinity, the diffused weights turn to be one everywhere, which reduces the non-uniform regularization to be uniform.

- Sparsity. When the time $t$ is small, the diffused weights are only nonzero around the control points. The sparsity of the weights will reduce the number of nonzero elements in the regularized deformation system and make the pre-factorization more efficient.

- Maximum principle. Due to the maximum principle of parabolic partial differential equations [25], the diffused weights of the control points are at their maximum. This property guarantees that the region around the control points will always receive stronger regularization.

- Shape-awareness. Since the diffused weights are directly solved from the diffusion equation on a triangular mesh, they are shape-aware. The weight of a point reflects the diffusion distance from this point to the control points instead of the Euclidean distance. Therefore ${ }^{306}$ it depends on the Riemannian metric of the shape and ${ }^{307}$ faithfully reflects the influence of the shape.

A naive choice of the weighting scheme is to simply define a310 Gaussian kernel or other radial basis functions centered at each ${ }_{311}$ control point and then blend them. However, the maximum ${ }^{312}$ principle does not always hold for this naive choice. When two 13 control points are close to each other, vertices between these two points may have higher weights than the control points. Another problem with this naive choice is that using Euclidean distance to compute the radial basis functions (RBF) is not suitable for freeform shapes. For example, if a control point is located at one tip of a hand-like shape, its influence cannot be evaluated using Euclidean distance since one handle only significantly controls one branch of the shape and Euclidean distance will confuse the branches. A correct choice of the distance measurement is to use geodesic or intrinsic distance. However, computing intrinsic distances on a freeform shape is no faster than solving a sparse linear system, which also prevents us from using radial basis functions to model the weights.

\subsection{Handle-specific Control}

In Sec. 3.2, we introduced our diffusion-based weighting scheme. With this model, we use the parameter $t$ to control the global scale of the non-uniform regularization. The diffused weights are shape-aware and each control point receives similar amount of regularization. In this section, we extend the diffusion model introduced in Sec. 3.2 to provide users with handle-specific control of the diffused weights. Specifically, users are allowed to control the range and magnitude of the weights around each handle. With the handle-specific control,

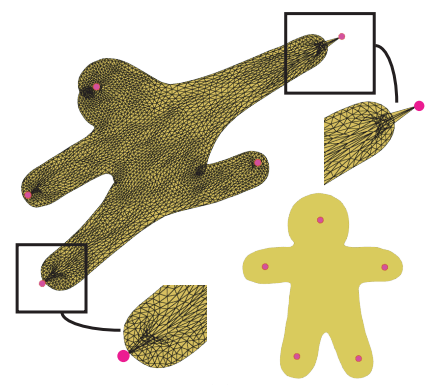

(a)

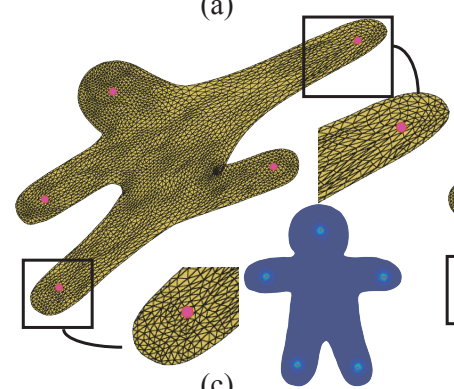

(c)

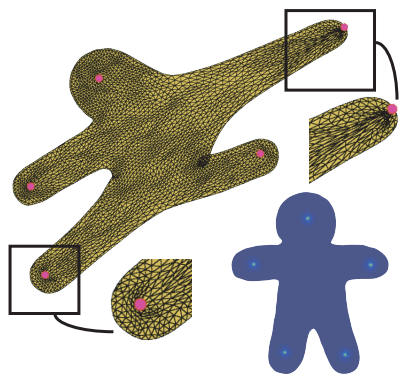

(b)

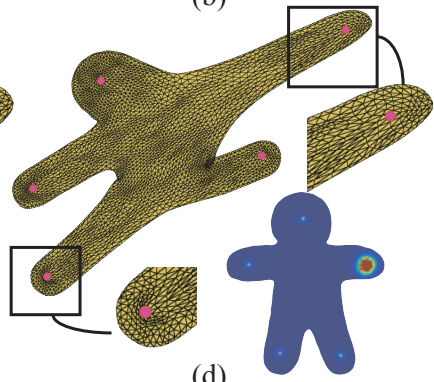

(d)

Figure 4. The deformation with no regularization (a), the non-uniformly regularized deformation without $(b, c)$ and with (d) handle specific control.

it is flexible to increase or decrease the amount of regularization for the region around each control handles. For example, as shown in Fig. 4, the non-uniformly regularized deformation without handle-specific control is either not strong enough to correct the artifacts around the right hand of woody or too strong to over-distort the left foot of woody. However, if users are allowed to only increase the range and magnitude of the regularization around the right hand region, the problem is solved.

Formally, we introduce two parameters $r_{j}, m_{j}$ for each control point, which respectively controls the local range and magnitude of the regularization around the jth control point. We first modify the traditional discrete Laplacian to reflect the effect of range control parameter $r_{j}$. In the diffusion equation, the speed of diffusion at the vertex $j$ is proportional to its Laplacian $\Delta \mathbf{x}_{j}$. Therefore, if we apply higher proportional coefficient on the control point $j$, the weights around it will diffuse faster and have larger range. Instead of formulating the proportional coefficient in (4), we directly introduce handle-specific parameters $\mathbf{r}=\left\{r_{1}, r_{2} \ldots, r_{n}\right\}$ to our discrete version (7) or (8) and let $L(\mathbf{r})$ denote the modified discrete Laplacian operator, which leads to solve

$$
(I-t L(\mathbf{r})) \mathbf{x}_{t}=\mathbf{x}_{0}
$$

or

$$
\left(I-t L(\mathbf{r})+\frac{t^{2}}{2} L^{2}(\mathbf{r})\right) \mathbf{x}_{t}=\mathbf{x}_{0} .
$$

Recalling that the discrete Laplacian

$$
L \mathbf{x}=\sum_{i=1}^{n} \sum_{j \in N(i)} w_{i j}\left(x_{i}-x_{j}\right)
$$

measures the integrated smoothness energy of the distribution, we will scale the smoothness energy at the vertex $i$ to control 


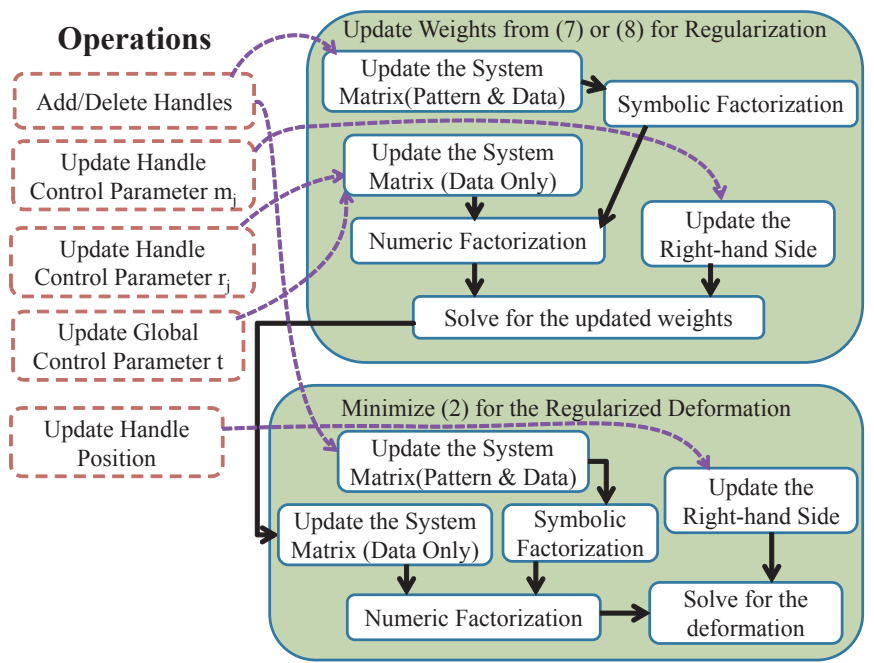

Figure 5. The schematic diagram for our variational deformation system with non-uniform regularization.

the speed of diffusion around the vertex $i$. So the matrix $L\left(\left\{r_{i}\right\}\right)$ works as

$$
L(\mathbf{r}) \mathbf{x}=\sum_{i=1}^{n} r_{i} \sum_{j \in N(i)} w_{i j}\left(x_{i}-x_{j}\right)
$$

where $N(i)$ is the one-ring neighboring vertices of the $i$ th vertex, $w_{i j}$ is the normalized cotangent weights of the edge $i j$ [23] and $r_{i}$ is the scaling coefficient. In our system, $r_{i}$ is set to 1 by default ${ }^{346}$ and users are allowed to increase or decrease the range of the weights around the control point $j$ by tuning $r_{j}$ up or down. $\operatorname{In}^{347}$ order to control the magnitude of the weights around the control ${ }^{348}$ point $j$, we simply modify the Dirichlet boundary condition that ${ }^{349}$ the $j$ th element of $\mathbf{x}_{t}$ is set to be $m_{j}$ for any $t$.

Note that it is also possible to use radial basis functions to ${ }^{351}$ control the range and magnitude. However, simply blending ${ }^{352}$ the radial basis functions at each handle is not suitable for our ${ }^{353}$ application, as discussed in Sec. 3.2.2.

In summary, the workflow of our deformation system is de- ${ }^{355}$ picted in Fig. 5. The diffused weights are computed by solv- ${ }^{356}$ ing a sparse linear system. Its sparsity pattern is static when ${ }^{357}$ users are moving the control point $j$, adjusting its local con- ${ }^{358}$ trol parameter $r_{j}, m_{j}$ or updating the global scale parameter $t .{ }^{359}$ So we compute the symbolic factorization of the sparse linear ${ }^{360}$ system once if no control points are added or deleted. When ${ }^{361}$ the parameters $r_{j}$ and $t$ are modified, the symbolic factorization ${ }^{362}$ is reused and we only need to run the numerical factorization. ${ }^{363}$ Because the parameter $m_{j}$ is modeled as boundary conditions ${ }^{364}$ and only appears at the right-hand side of the linear system, no ${ }^{365}$ re-factorization is required if only $m_{j}$ is updated. After the dif- ${ }^{366}$ fused weights are obtained, we proceed to minimize (2) for the ${ }^{367}$ deformed shape. Note that the regularized linear systems de- ${ }^{368}$ rived from minimizing (2) also has static sparsity patterns if the ${ }^{369}$ structure of the control points is fixed. But the coefficients of ${ }^{370}$ the sparse matrix are dependent on the diffused weights. There- ${ }^{371}$ fore, we also symbolically factorize the system from (2) once ${ }^{372}$ and reuse it to accelerate its numerical factorization after each ${ }^{373}$ edit of the diffused weights.

\begin{tabular}{|c|c|c|c|c|c|c|}
\hline Figure & $\# \mathrm{~V}$ & $\# \mathrm{~F}$ & $D_{\max }$ & $D_{\text {ave }}$ & $D_{d e v}$ & $\overline{\text { Dist }}$ \\
\hline 6(a)left & & & 11.29 & 0.14 & 0.53 & \\
\hline 6(a)middle & 432 & 715 & 3.77 & 0.28 & 0.38 & 0.00168 \\
\hline $6(a) r$ & & & .38 & .22 & 0.34 & 0.00095 \\
\hline 6(b)l & & & 8.59 & .29 & 0.57 & \\
\hline 6(b)n & 1074 & 1923 & 1.73 & 0.52 & 0.32 & 0.013 \\
\hline 6(b)right & & & 1.64 & 0.43 & 0.34 & 0.010 \\
\hline 6(c)le & & & 11.87 & 0.01 & 0.31 & - \\
\hline $6(\mathrm{c}) 1$ & 3208 & 5981 & 1.50 & 0.18 & 0.25 & $4.695 e-4$ \\
\hline 6(c) & & & 1.69 & 0.14 & 0.23 & $3.044 \mathrm{e}-4$ \\
\hline 7(a) & & & 13.06 & 0.32 & 0.77 & \\
\hline 7 (a) & 630 & 1042 & 3.64 & 0.48 & 0.49 & 0.0097 \\
\hline $7(a)$ & & & 2.57 & 0.43 & 0.45 & 0.0083 \\
\hline $7(\mathrm{~b}) \mathrm{le}$ & & & 7.11 & 0.24 & 0.55 & - \\
\hline 7(b)middle & 630 & 1042 & 1.67 & 0.40 & 0.31 & 0.0096 \\
\hline 7(b)right & & & 1.20 & 0.35 & 0.29 & 0.0082 \\
\hline 7(c)left & & & 1.86 & 0.06 & 0.15 & \\
\hline 7(c) & 630 & 1042 & 0.59 & 0.10 & 0.12 & 0.0117 \\
\hline 7 (c)right & & & 0.55 & 0.10 & 0.12 & 0.0115 \\
\hline
\end{tabular}

Table 1. Model statistics. \#V and \#F are the number of the vertices and faces. The maximum $D_{m} a x$, average $D_{a} v e$ of the distortion per face and its standard deviation $D_{d} e v$ are used to characterize the quality of the deformation. Dist measures the root mean square error of the regularized deformation to the deformed shape without regularization.

\section{Results}

We show the deformation results with our non-uniform regularization in this section. Note that we do not directly suggest the best trade-off between the shape smoothness and distortion. Instead, we provide a method that allows users to freely control the regularization around each control point and explore the locally regularized deformation accordingly.

We experiment our non-uniform regularizer with various models and different distortion measurement, comparing with the results from no regularization and uniform regularization [4], as shown in Fig. 6 and Fig. 7. Please note that for nonlinear and nonconvex deformation energies such as ARAP, the results may get stuck in local optimum, even if our regularizer is quadratic and the approximate diffused weights are solved from linear equations. In the experiment, we directly use the rest shape as the initial guess. In Fig. 6 and Fig. 7, both texture mapping and color-coded energy maps are used to highlight the distortion, where red and blue depict high and low distortion respectively in the color map. We also list the distribution of the distortion per face in Tab. 1. From the images and the statistics, we see that our non-uniform regularization efficiently smoothes out the discontinuity artifact while preventing higher distortion of the elements far away from the control points. From the lower distance to the deformed shape without regularization, we see that our technique only locally corrects the artifacts around the control points and better keeps the deformation with no regularization.

Note that, different from the color-coded energy maps in Fig. 6, the color maps in the rightmost figures of Fig. 7 help vi- 
(a)
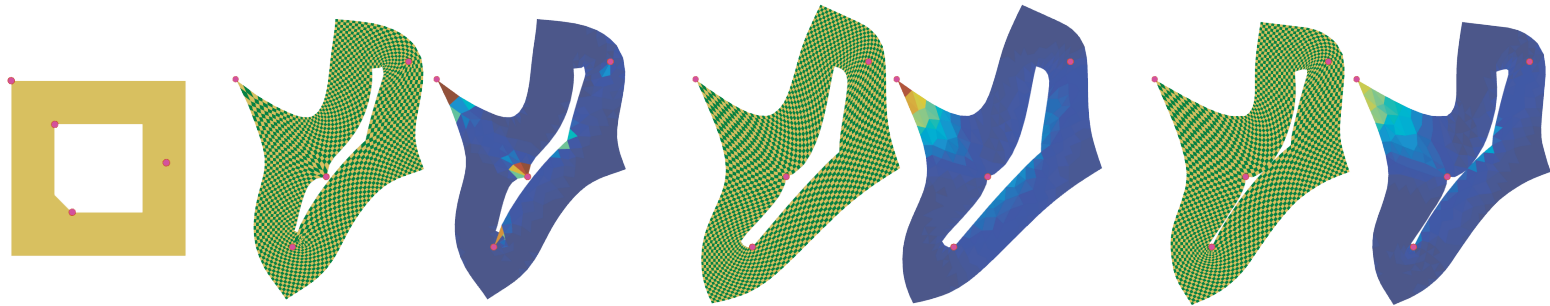

(b)

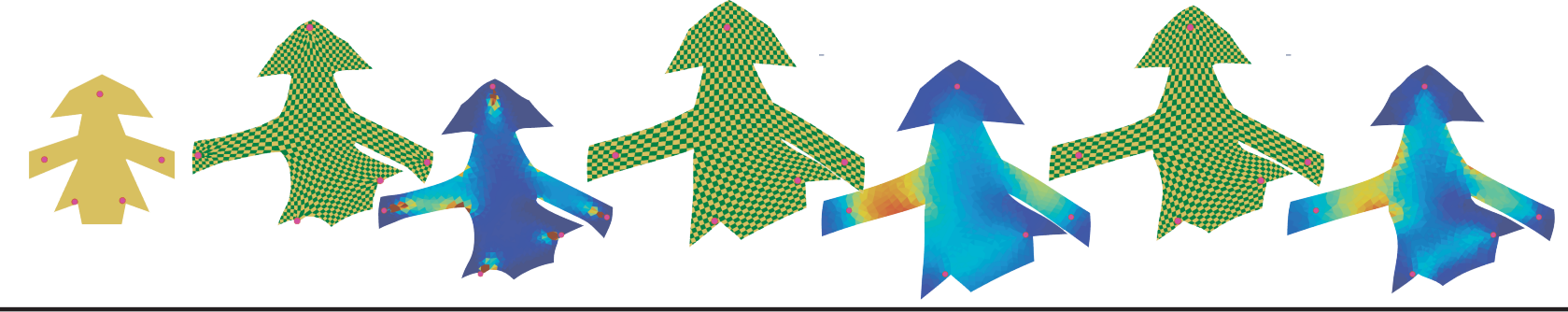

(c)
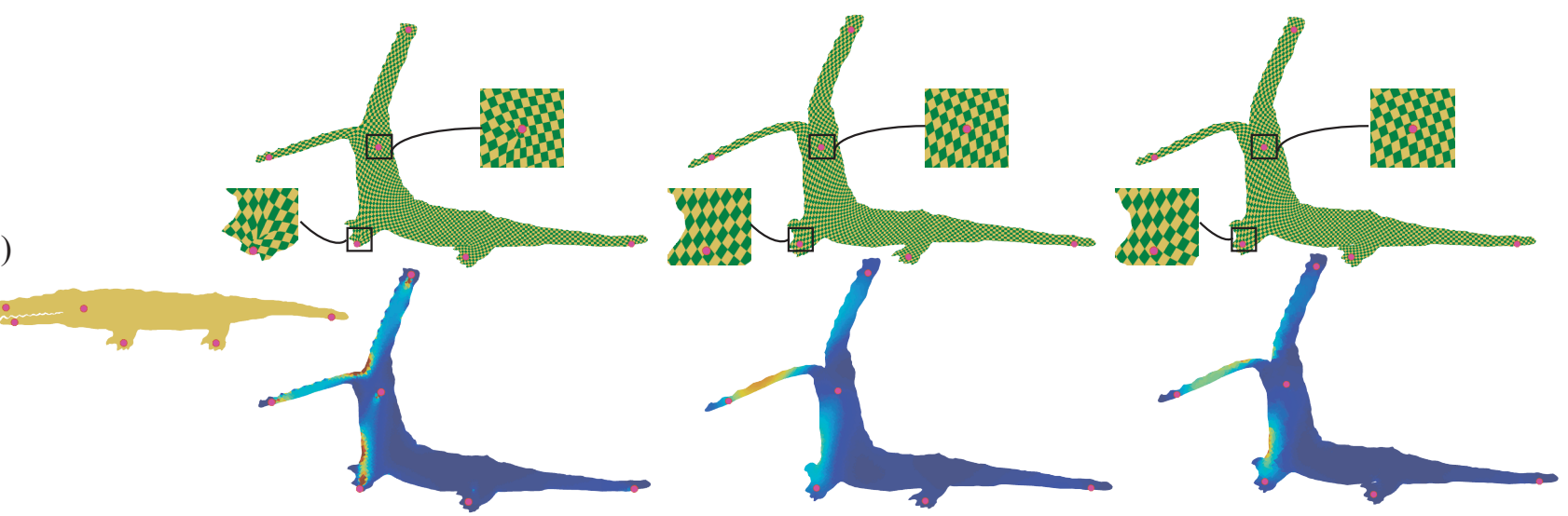

Figure 6. From left to right: the rest shape with control points, the deformed shape without, with uniform and with non-uniform regularization. The ARAP energy is used to measure the distortion here.

(a)

ARAP
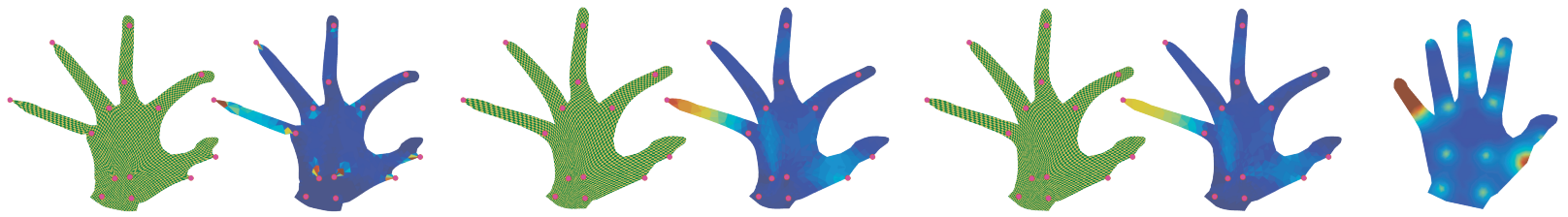

(b)

IARAP
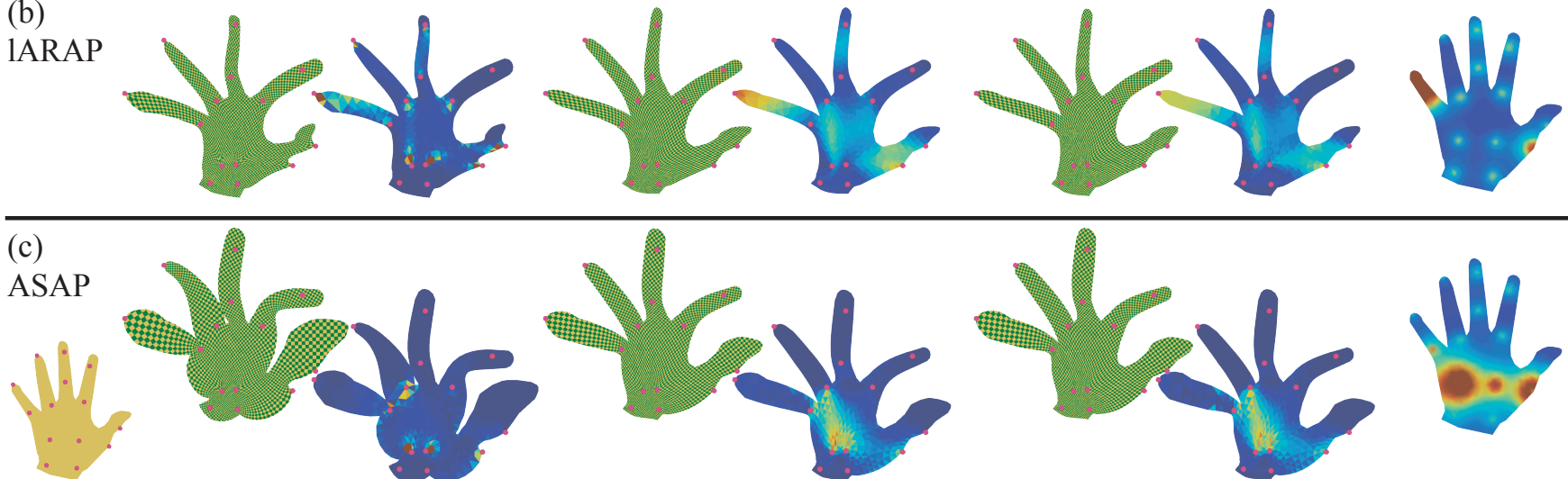

Figure 7. From left to right: the deformed shape without, with uniform and with non-uniform regularization. The rightmost figures show the distribution of the diffused weights. The leftmost subplot shows the rest shape and the control points. The deformed shape are computed by minimizing the ARAP energy (a), the linearized ARAP (lARAP) energy (b) or the ASAP energy (c). 


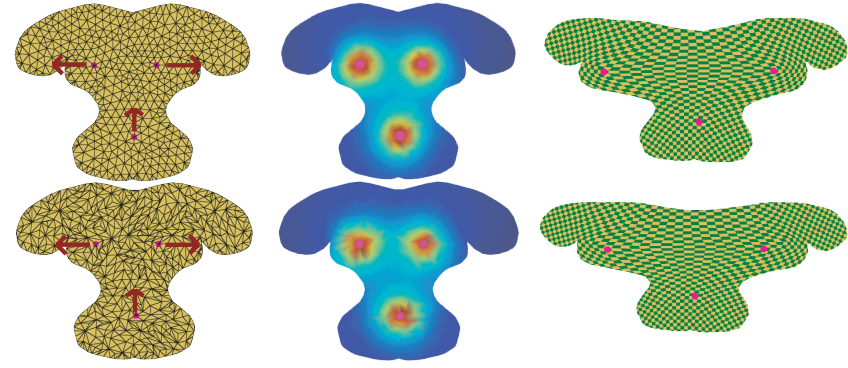

Figure 8. The regularized deformation of the same shape with different triangulation. From left to right: the triangulation of the rest shape with their control points, the diffused weights, and the regularized deformation.

sualize the diffused weights. Those figures show that users are allowed to control both the magnitude and range of the regularization for each control point. Besides, they also demonstrate the shape-awareness of our diffusion-based weighting scheme, as discussed in Sec. 3.2.2.

The diffused weights are not quite sensitive to meshes with ${ }^{398}$ different triangulation, as shown in Fig. 8. We randomly flip ${ }^{399}$ the edges between pairs of neighboring triangles shown in the ${ }^{400}$ upper row and generate an irregularly triangulated mesh shown ${ }^{401}$ in the lower row. By running our algorithm with the same defor- ${ }^{402}$ mation configuration, we get similar distribution of the weights ${ }^{403}$ and regularized deformation. Note that the discrete Laplacian ${ }^{404}$ we use [23] is shown to be positive semi-definite in [26]. ${ }^{405}$ Therefore, for the purpose of computing diffusion, our method ${ }^{406}$ does not induce numerical instability on irregular meshes. $\quad{ }_{407}$

We then test the diffusion-based weighting scheme on $\mathrm{a}^{408}$ mesh with different resolutions. We will have to increase the ${ }^{409}$ time parameter $t$ if a similar regularized deformation is required ${ }^{410}$ on a dense mesh, as shown in Fig. 9. This corresponds to the ${ }^{411}$ observation in [27] that the solution of the discretized diffusion ${ }^{412}$ equation is resolution-dependent. The solved weights at a large ${ }^{413}$ time $t$ can be well approximated by the solution of the diffu- ${ }^{414}$ sion equation at a small time $t$ on a coarse mesh. Vaxman et $t^{415}$

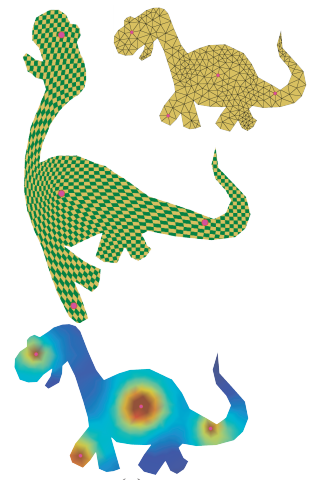

(a)

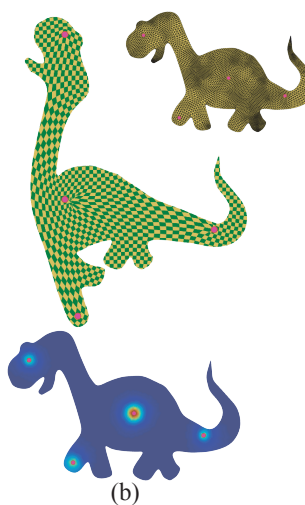

(b)

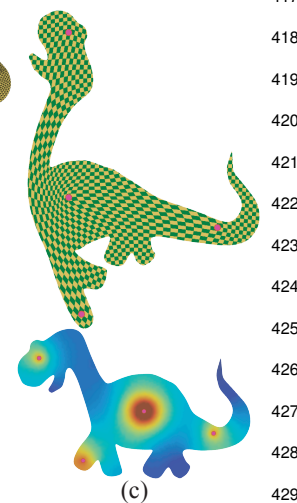

(c)
The deformed coarse mesh with handle specific control $\left\{m_{j, r}\right.$ time $t$; (b) the deformed dense mesh with the same deformation setting as (a), which does not fully correct the discontinuity artifacts; (c) the deformed dense ${ }^{432}$ mesh with larger time $t$ to reproduce the similar deformed shape as (a). Two ${ }^{433}$ subplots at the top of (a) and (b) show the control points on the rest coarse and $\mathrm{d}_{434}$ dense mesh.
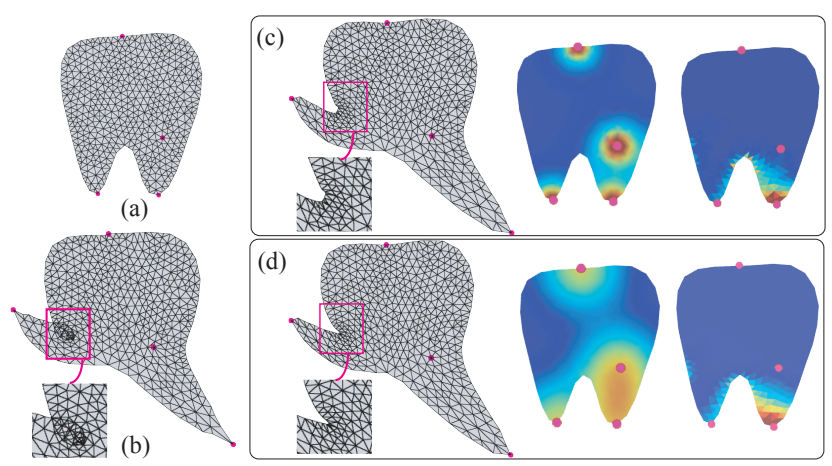

Figure 10. (a)The rest mesh and its control points. (b)The ARAP deformation without regularization. (c) The non-uniformly regularized ARAP deformation with the weights generated from diffusion. (d) The non-uniformly regularized ARAP deformation with the RBF-based weights. In the subfigures of (c) and (d), we show the deformed shapes, the distribution of the weights and the ARAP distortion energy from left to right. The subplots show that the regularization eliminates the foldover introduced by ARAP.

al [14] use this property to accelerate the computation of diffusion equation by building a hierarchy of meshes with various resolution. We do not use this method to compute the diffused weights because it also takes time to prepare the mesh hierarchy. Since it is not straightforward to predict the correct time $t$ with respect to the mesh resolution, we provide an interface that allows user to tune up and down the parameters $\left\{m_{j}, r_{j}\right\}$ and $t$ until the user is satisfied with deformation or the diffused weights. The user interface is intuitive because the range of the diffused weights around the control point $i$ is monotonically increasing with $t$ or $r_{i}$ increasing. Besides, our diffusion model only requires to solve one sparse linear system, which interactively gives feedbacks to the user.

We also compare our diffusion-based weighting scheme with RBF-based weighting scheme, which is another option to create handle-aware weights. As discussed in Sec.3.2.2, RBFbased weighting scheme accumulates the weights around each handle and thus does not hold the maximum principle. Therefore, it may over-regularize the region between two neighboring control points, which results in higher distortion comparing with our weighting scheme, as shown in the color maps in Fig. 10(d). Besides, RBF-based weighting scheme requires the prepared geodesic distances to make the weghts shape-aware. We time the geodesic distance computation between all points and the control points by using the library provided in [28]. For the model shown in Fig. 10 with 536 vertices and 960 faces, it costs around $28 \mathrm{~ms}$ to compute the necessary distances, while our diffusion-based weighting scheme only costs $2 \mathrm{~ms}$ to precompute the factorization and less than $1 \mathrm{~ms}$ to solve for the weights. Although new algorithms for computing geodesic distances whose performance reaches more than 4 times faster than [28] are developed recently, they are still not as fast as solving one sparse linear system to obtain the weights.

Our method can be applied to obtain smooth deformation. Pure variation deformation such as ARAP only focuses on minimizing the distortion and is prone to introduce discontinuity near the control points ( Fig. 11(a)). The best way to get smooth 


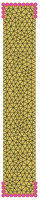

(a)

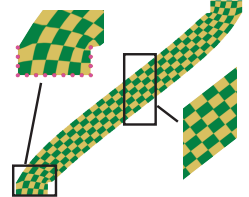

(b)

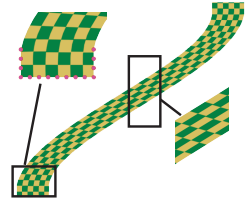

(c)

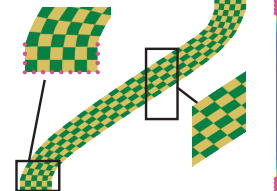

(d)

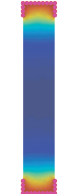

(e)
Figure 11. (a)The rest mesh and its control points. (b)The ARAP deformation without regularization. (c) The uniformly regularized ARAP deformation. (d)The non-uniformly regularized ARAP deformation. (e) The diffused weights for regularization.

deformation is to use the regularizer proposed in [4] and set $\beta=1$ in the uniform regularization (2). However, since no distortion energy is penalizing the deformation when $\beta=1$, the elements will be highly distorted. Therefore, by tuning $\beta$ in $[0,1]$, we are searching for a trade-off between the lower distortion and better smoothness. One typical result is shown in Fig. 11(b). Note the volume loss and highly distorted elements at the middle of the bar are unavoidable. Our non-uniform regularization strives to fix the nonsmoothness around the control points while keep the distortion energy low far away from the control points. It provides a better trade-off of the distortion and smoothness, as shown in Fig. 11(c).

Our method also works for 3D thin shell and solid deformation, as shown in Fig. 12. The only modification we made is to replace the $2 \mathrm{D}$ version of the discrete Laplacian in $(7)$ or $(8)^{480}$ with discrete Laplacian-Beltrami operator or the $3 \mathrm{D}$ version of ${ }^{481}$ the discrete Laplacian. The spikes are all removed after regu- ${ }^{482}$ larization. Note that our non-uniform regularization introduces ${ }^{483}$ less global distortion, e.g., the global bump on the front of the ${ }^{484}$ beetle model is less observable when using our non-uniform ${ }^{485}$ regularization, in comparison with the uniformly regularized ${ }^{486}$ shape. For the rocker-arm model, our method creates a de- ${ }^{487}$ formed model with less global stretch. The deformed chair ${ }^{488}$ model with uniform regularization makes the leg less straight ${ }^{489}$ while our method alleviates this problem and generates a result ${ }^{490}$ with less distortion.

To evaluate the computational time, all the experiments are ${ }^{492}$ performed on a single CPU thread with $2.5 \mathrm{GHz}$. Because we $\mathrm{e}^{493}$ only need to solve two sparse linear systems, the diffused weights and the deformation using quadratic distortion measurements are all computed at an interactive rate for meshes with up to $5 \mathrm{~K}$ vertices, if no handles are added or removed. If only the control points are moving, our system supports editing shapes with up to $10 \mathrm{k}$ vertices interactively. As claimed in Sec.3.2.1, we generally solve eqn. (8) for better precision although it is slower than solving eqn. (7). Because for meshes with moderate number of vertices, the computational cost does not make noticeable differences to the users. For example, for the car body model in Fig. 12 with 984 vertices and 1759 faces, the statistics for the time of factorizing eqn. (7), factorizing eqn. (8), solving eqn. (7) and solving eqn. (8) are $8 \mathrm{~ms}, 32 \mathrm{~ms}, 1 \mathrm{~ms}$ and $2 \mathrm{~ms}$, respectively. The corresponding data increase to $1.13 \mathrm{~s}, 8.5 \mathrm{~s}$, $11 \mathrm{~ms}$ and $30 \mathrm{~ms}$ for the rocker-arm model in Fig. 12 with 3,696 vertices and 13,088 tetrahedrons. We also report here the computational time for a larger model, the chair model in Fig. 12

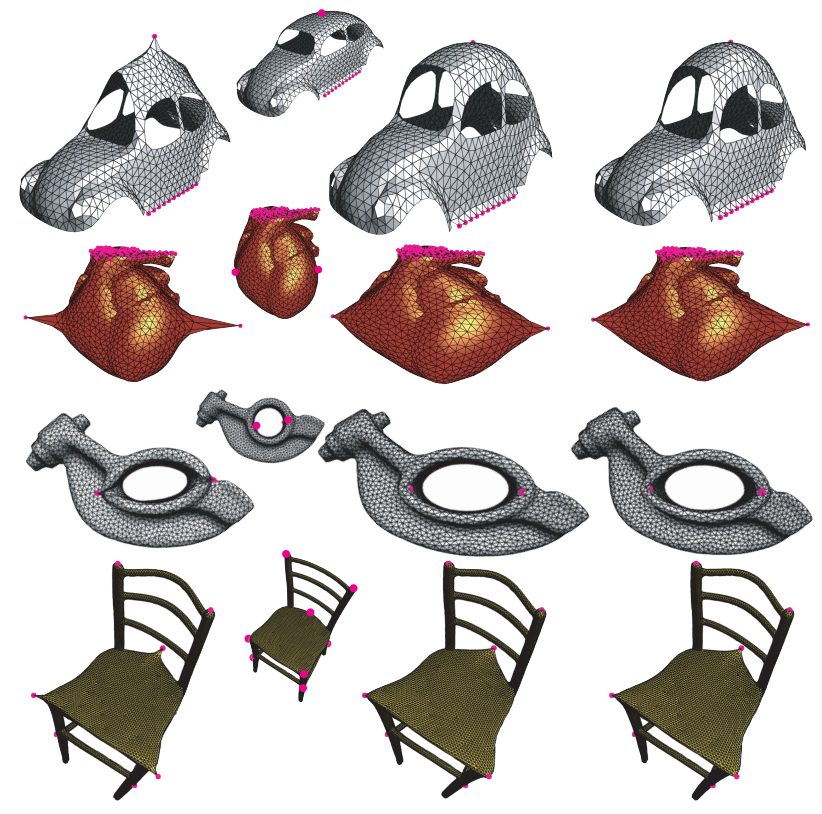

Figure 12. Deforming 3D thin shell and solid models with ARAP(left), uniformly regularized ARAP(middle right) and non-uniformly regularized ARAP(right). The undeformed shapes and control points are shown in the middle-left subfigures.

with 21,342 vertices and 92,500 tetrahedrons. It costs $9.04 \mathrm{~s}$ and 52.8 s to factorize eqn. (7) and eqn. (8), respectively, and solving them costs $0.06 \mathrm{~s}$ and $0.17 \mathrm{~s}$, respectively.

At last, we discuss about typical failure cases of our method. ARAP deformation without regularization may introduce nondiffeomorphic mapping. By applying the proposed regularization, it can be avoided as the distortion of the deformed shape is reducing, as highlighted in the subplots in Fig. 10. However, as shown in the lower row of Fig. 13, when the deformation goes extremely large, even with a strong regularizer, it is still hard to eliminate all the inverted faces. This is because the expected deformation without inversion is highly nonlinear, it is difficult to produce highly nonlinear deformation with quadratic regularizers.

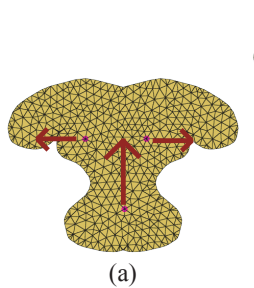

(a)

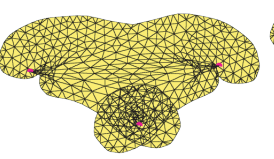

(b)

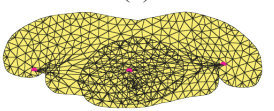

(d)

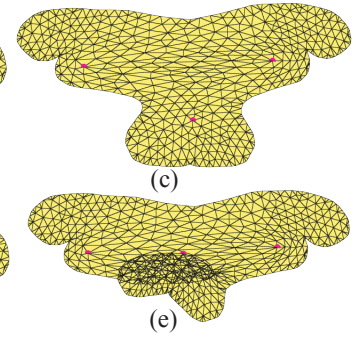

Figure 13. (a) The editing path of the control points on the rest shape. As the lower control point goes up, the deformed shape with our proposed regularization (c) is free of foldover, comparing with the ARAP deformation without regularization (b). When the lower control point keeps moving up, our method (e) does not guarantee to produce foldover-free results, although its deformation energy is still lower than the deformation without regularization (d). 


\section{Conclusion}

In this work, we introduce a non-uniform regularization tech55 nique for variational shape deformation. We use diffusion-based ${ }^{552}$ techniques to generate a smooth scalar field and users are al- ${ }_{554}^{553}$ lowed to control the magnitude and range of the regularization.555 With our technique, it is possible to interactively control the de-556 formation with no noticeable discontinuity artifacts, while the ${ }_{558}^{557}$ global shape distortion is kept lower.

We extend the traditional regularization techniques to bring ${ }_{560}$ more flexible control of the regularizer. The flexibility is at ${ }^{561}$ the price that users are required to adjust more parameters. $\mathrm{An}^{562}$ interesting direction for future work is to automatically select ${ }_{564}$ parameters for any specified deformation. We approximate the 565 diffusion equation with one linear equation in this work, which ${ }^{566}$ does not produce the exact solution on shapes. It is still worth ${ }_{568}^{567}$ studying how to efficiently solve the diffused weights with highe ${ }_{69}$ precision. Besides, our method works under the assumption 570 that the smoothness of the distortion map around control points ${ }^{571}$ well follows the diffusion model. There is no theoretical guar- ${ }_{573}^{572}$ antee about this assumption and it is essential to continue a the- 574 oretical study to model the discontinuity artifacts. In addition, 575 since bijectivity is not explicitly modeled as a hard constraint ${ }^{576}$ in the deformation energy or the regularizer, the regularized ${ }_{578}^{577}$ deformation is not guaranteed to be bijective even if a strong ${ }_{579}$ quadratic regularizer is applied. We will keep exploring effec-580 tive regularization techniques to suppress the inverted elements ${ }_{588}^{581}$ under extreme deformation.

\section{Acknowledgements}

The authors would like to thank anonymous reviewers fors their constructive comments. The beetle, rocker-arm and chair ${ }^{589}$ model is used courtesy of Aim@Shape and the alligator and ${ }_{591}^{590}$ dino meshes are remeshed from [4]. This work has been sup- ${ }_{592}$ ported by the National Science Foundation of China(NSFC) un-593 der Grants No.61502096, 61502240 and 61304205, the Natural ${ }^{594}$ Science Foundation of Jiangsu Province of China under Grants ${ }_{596}^{595}$ No.BK20150634, BK20160697, BK20141002, the Fundamen- ${ }_{597}^{596}$ tal Research Funds for the Central Universities and the Open598 Fund of Jiangsu Province Key laboratory of Remote Measuring ${ }^{599}$ and Control.

\section{References}

[1] M. Botsch, O. Sorkine, On linear variational surface deformation meth- ${ }^{60}$ ods, IEEE Transactions on Visualization and Computer Graphics 14 (1) 607 (2008) 213-230.

[2] S. Bouaziz, M. Deuss, Y. Schwartzburg, T. Weise, M. Pauly, Shape-up: Shaping discrete geometry with projections, Computer Graphics Forum 31 (5) (2012) 1657-1667.

[3] Z. Levi, D. Zorin, Strict minimizers for geometric optimization, ACM Trans. Graph. 33 (6) (2014) 185:1-185:14.

[4] J. Martinez Esturo, C. Rossl, H. Theisel, Smoothed quadratic energies on meshes, ACM Trans. Graph. 34 (1) (2014) 2:1-2:12.

[5] A. Jacobson, I. Baran, L. Kavan, J. Popović, O. Sorkine, Fast automatic skinning transformations, ACM Transactions on Graphics (proceedings of ACM SIGGRAPH) 31 (4) (2012) 77:1-77:10.

[6] Y. Wang, A. Jacobson, J. Barbic, L. Kavan, Linear subspace design for real-time shape deformation, ACM Trans. Graph. 34 (4).
[7] X.-M. Fu, Y. Liu, B. Guo, Computing locally injective mappings by advanced mips, ACM Trans. Graph. 34 (4) (2015) 71:1-71:12.

[8] Y. Lipman, Bounded distortion mapping spaces for triangular meshes, ACM Trans. Graph. 31 (4) (2012) 108:1-108:13.

[9] S. Z. Kovalsky, N. Aigerman, R. Basri, Y. Lipman, Controlling singular values with semidefinite programming, ACM Trans. Graph. 33 (4) (2014) 68:1-68:13.

[10] C. Schüller, L. Kavan, D. Panozzo, O. Sorkine-Hornung, Locally injective mappings, Computer Graphics Forum (proceedings of EUROGRAPHICS/ACM SIGGRAPH Symposium on Geometry Processing) 32 (5) (2013) 125-135.

[11] Y. Kozlov, J. M. Esturo, H.-P. Seidel, T. Weinkauf, Regularized harmonic surface deformation, (2014). arXiv:1408.3326.

[12] M. Desbrun, M. Meyer, P. Schroder, A. H. Barr, Implicit fairing of irregular meshes using diffusion and curvature flow, in: Proceedings of the 26th Annual Conference on Computer Graphics and Interactive Techniques, SIGGRAPH '99, 1999, pp. 317-324.

[13] J. Sun, M. Ovsjanikov, L. Guibas, A concise and provably informative multi-scale signature based on heat diffusion, Computer Graphics Forum 28 (5) (2009) 1383-1392.

[14] A. Vaxman, M. Ben-Chen, C. Gotsman, A multi-resolution approach to heat kernels on discrete surfaces, ACM Trans. Graph. 29 (4) (2010) 121:1-121:10.

[15] K. Crane, C. Weischedel, M. Wardetzky, Geodesics in heat: A new approach to computing distance based on heat flow, ACM Trans. Graph. 32 (5) (2013) 152:1-152:11.

[16] F. De Goes, S. Goldenstein, L. Velho, A hierarchical segmentation of articulated bodies, Computer Graphics Forum 27 (5) (2008) 1349-1356.

[17] M. Ovsjanikov, Q. Mrigot, F. Memoli, L. Guibas, One point isometric matching with the heat kernel, Computer Graphics Forum 29 (5).

[18] H. DU, T. YOO, H. QIN, Pde-based medial axis extraction and shape manipulation of arbitrary meshes, Journal of Systems Science and Complexity 21 (4) (2008) 609-625.

[19] L. Liu, L. Zhang, Y. Xu, C. Gotsman, S. J. Gortler, A local/global approach to mesh parameterization, Computer Graphics Forum 27 (5) (2008) 1495-1504.

[20] O. Sorkine, M. Alexa, As-rigid-as-possible surface modeling, in: Proceedings of EUROGRAPHICS/ACM SIGGRAPH Symposium on Geometry Processing, 2007, pp. 109-116.

[21] T. Igarashi, T. Moscovich, J. F. Hughes, As-rigid-as-possible shape manipulation, ACM Trans. Graph. 24 (3) (2005) 1134-1141.

[22] M. Botsch, L. Kobbelt, M. Pauly, P. Alliez, B. Lévy, Polygon Mesh Processing, AK Peters / CRC Press, 2010.

[23] M. Meyer, M. Desbrun, P. Schroder, A. H. Barr, Discrete differentialgeometry operators for triangulated 2-manifolds, in: Visualization and Mathematics III, Springer-Verlag, 2003, pp. 35-57.

[24] M. Chuang, M. Kazhdan, Interactive and anisotropic geometry processing using the screened poisson equation, ACM Trans. Graph. 30 (4) (2011) 57:1-57:10.

[25] M. H. Protter, H. F. Weinberger, Maximum Principles in Differential Equations, Springer, 1999

[26] M. Wardetzky, S. Mathur, F. Kälberer, E. Grinspun, Discrete laplace operators: No free lunch, in: Proceedings of the Fifth Eurographics Symposium on Geometry Processing, 2007, pp. 33-37.

[27] F. Memoli, Spectral gromov-wasserstein distances for shape matching, in: Proceedings of Workshop on Non-Rigid Shape Analysis and Deformable Image Alignment, 2009.

[28] V. Surazhsky, T. Surazhsky, D. Kirsanov, S. J. Gortler, H. Hoppe, Fast exact and approximate geodesics on meshes, ACM Trans. Graph. 24 (3) (2005) 553-560. 